\title{
Multichannel surface clutter suppression: East Antarctica P-band SAR ice sounding in the presence of grating lobes
}

\author{
David BEKAERT, ${ }^{1 *}$ Nicolas GEBERT, ${ }^{1}$ Chung-Chi LIN, ${ }^{1}$ Florence HÉLIÈRE, ${ }^{1}$ \\ Jørgen DALL, ${ }^{2}$ Anders KUSK, ${ }^{2}$ Steen SAVSTRUP KRISTENSEN ${ }^{2}$ \\ ${ }^{1}$ Earth Observation Projects Department, European Space Research and Technology Centre (ESTEC), Noordwijk, \\ The Netherlands \\ E-mail: eedpsb@leeds.ac.uk \\ ${ }^{2}$ National Space Institute, Technical University of Denmark (DTU), Lyngby, Denmark
}

\begin{abstract}
Ice sounding with radar is a well-established technique for the retrieval of ice depth, and provides information on ice structures and layering. Airborne radar ice sounders suffer from off-nadir surface clutter that masks the signal from bedrock and ice layers with unwanted but simultaneously received surface reflections. This is of importance for future satellite ice-sounding missions, as the spaceborne geometry leads to strong surface clutter even for deep subsurface returns. This paper presents analysis and comparison of different clutter-suppression techniques applied to data acquired with the European Space Agency's P-band POLarimetric Airborne Radar Ice Sounder (POLARIS). The $4 \mathrm{~m}$ long antenna of POLARIS enables simultaneous reception of up to four across-track channels. It was operated in 2011 over Antarctica at a high flight altitude of $3200 \mathrm{~m}$. Different coherent weighting techniques of the receive channels were used to suppress the surface 'clutter'. However, with a channel spacing of 1.4 times the wavelength, the grating lobe imposes a limitation to the off-nadir angular range in which clutter can be effectively attenuated. Results of ice sounding over Jutulstraumen glacier are described, where we demonstrate a clutter suppression of up to $10 \mathrm{~dB}$.
\end{abstract}

KEYWORDS: Antarctic glaciology, ground-penetrating radar, ice streams, remote sensing

\section{INTRODUCTION}

Understanding the role of the cryosphere in climate change is one of the key elements of the European Space Agency's (ESA's) Living Planet program (ESA, 2013). Radar ice sounding, with its ability to provide two-dimensional characteristics of the major ice sheets, represents a very important observational tool, which will improve understanding of the history and evolution of ice sheets.

\subsection{Radar ice sounding from space}

Use of long-range aircraft flying at high altitude is desirable for covering the Earth's ice sheets, due to their large area requiring large-scale spatial coverage. A spaceborne ice sounder would provide the additional benefit of global coverage and observations of uniform quality. Attenuation of electromagnetic waves in ice is small for frequencies up to $\sim 1 \mathrm{GHz}$ (Fujita and others, 2000). Nevertheless, the offnadir surface scattering and volume scattering due to ice inclusions and inhomogeneities monotonically increase with frequency. In particular, ice surfaces that are rough compared to the wavelength can lead to unwanted off-nadir surface scattering ('clutter') of considerable strength (Jezek and others, 2011).

For this reason, current airborne sensors for ice sounding operate at low frequencies (e.g. 60 or $150 \mathrm{MHz}$ (Peters and others, 2007; Jezek and others, 2011)), penetrating deep into the ice and allowing imaging of the bedrock underneath. Unfortunately, such low frequencies require very large antenna dimensions for a spaceborne instrument, which

*Present address: School of Earth and Environment, University of Leeds, Leeds, UK. cannot be easily accommodated on a spacecraft within a launcher fairing. The limitation on the allowable antenna size leads to a preference for a radar sounder operating at a higher frequency. The lowest frequency range allocated by International Telecommunications Union regulations for active sensing from space is $432-438 \mathrm{MHz}$ (P-band). P-band potentially represents a good compromise between the necessary antenna dimensions and achieving the required gain and penetration capability (Jezek and others, 2006). Nevertheless, at P-band the surface clutter masking the subsurface echoes represents a major challenge for a radar operated from an Earth-orbiting satellite at an altitude of several hundred kilometers (Jezek and others, 2011), suggesting the need for sophisticated signal-processing techniques to suppress surface clutter (Dall and others, 2010).

The large antenna area (sought to provide high antenna gain) needs to be subdivided into individual apertures ('channels') during reception, in order to allow for surfaceclutter-suppression techniques. A minimum number of receive apertures is desired to keep system complexity at a minimum. For a given antenna length, this means maximizing the sub-aperture length, which is then likely to amount to several wavelengths, giving rise to substantial grating lobes, even in the case of sensors flying at high altitude. Consequently, the applicability of clutter-suppression techniques in the presence of grating lobes enables a further reduction of the number of sub-apertures, thereby simplifying the system design.

\subsection{POLARIS instrument and campaigns}

The P-band $(435 \mathrm{MHz})$ POLarimetric Airborne Radar Ice Sounder (POLARIS), developed for ESA by the Technical University of Denmark (DTU), was operated over Greenland 


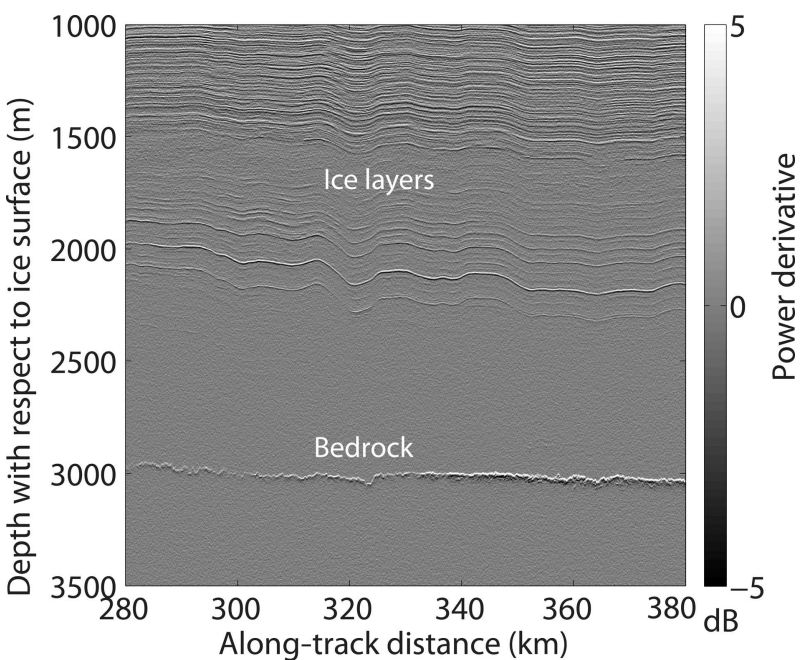

Fig. 1. Ice-sounding result, demonstrating the capabilities of POLARIS (Greenland 2009).

in 2008 and 2009 in a single-aperture and quad-polarized configuration, having an antenna length of $2 \mathrm{~m}$ (Dall and others, 2010). The 2009 campaign included flights over the NEEM (North Greenland Eemian Ice Drilling project) drill sites in directions parallel and orthogonal to the ice flow, for studying the birefringence. POLARIS imaged the bedrock at $3000 \mathrm{~m}$ depth (Dall and others, 2010), demonstrating the high sensitivity of the instrument and confirming the suitability of P-band for ice sounding (Fig. 1).

Subsequently, POLARIS was enhanced with a larger antenna of $4 \mathrm{~m}$ length (Fig. 2), with eight radiating units grouped into four independent phase centers, enabling simultaneous reception, digitization and storage of up to four channels on board. While operating POLARIS in the multichannel mode during the 2011 Antarctica campaign, a cabling error introduced a phase shift of $180^{\circ}$ between individual receive channels. As each channel is acquired individually, this does not affect the receive patterns, but a quasi-null was generated, with a clearly reduced gain in the transmit antenna pattern at boresight (Fig. 3). Though deep, the erroneous null is very narrow and only partly masks the angular range over which the echo from nadir is received. As a consequence, the sensitivity towards the subsurface reflections is significantly reduced (i.e. $>20 \mathrm{~dB}$ ), making the suppression of surface clutter even more important. A detailed description of POLARIS is given by Dall and others (2010), and Table 1 summarizes the key parameters of the multichannel POLARIS configuration.

Table 1. Key parameters of POLARIS operated over Jutulstraumen

\begin{tabular}{lc}
\hline Parameter & Value \\
\hline Center frequency & $435 \mathrm{MHz}$ \\
Transmitted bandwidths & $85 / 30 / 6 \mathrm{MHz}$ \\
Pulse duration & $2 / 15 / 15 \mu \mathrm{s}$ \\
Polarization & $\mathrm{VV}$ \\
Number of channels & 4 \\
Number of radiating elements & 8 \\
Element cross-track spacing (w.r.t. center) & $0.48 \mathrm{~m}$ \\
Element size & $0.408 \mathrm{~m} \times 0.408 \mathrm{~m}$
\end{tabular}

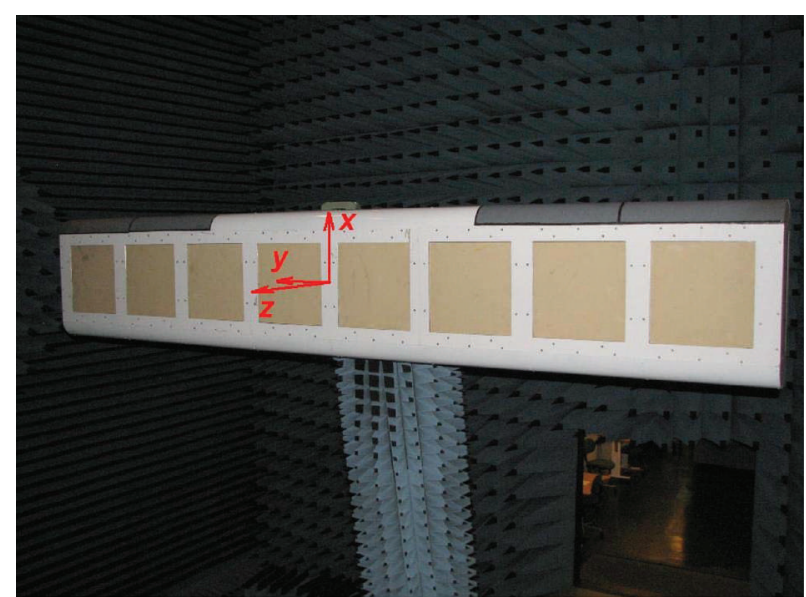

Fig. 2. Upgraded POLARIS antenna during radio-frequency measurement at the DTU-ESA facility, courtesy of DTU.

All acquired POLARIS campaign data are available at the ESA Earth Observation Campaign website (http://earth.esa. int/). An overview of the multichannel flight tracks acquired over Jutulstraumen glacier, Antarctica, is provided in Figure 4. In total, four tracks were flown. Tracks Jsew1 and Jswe1 were acquired approximately perpendicular to the glacier in opposite directions, while tracks Jsns1 and Jsns2 were acquired in the direction parallel to the glacier, respectively flying downstream on the glacier edge and upstream on the glacier center line. Based on bedrock/water reflections in the clutter-prone region, tracks Jsew1 and Jsns2 were selected to demonstrate clutter suppression for this paper. Both tracks have low across-track topographic variations.

\subsection{Processing}

Processing begins by focusing the synthetic aperture radar (SAR) image, i.e. compression in both range and azimuth, for each of the individual channels. Azimuth focusing depends on the ice depth, as the along-track phase history of a target varies with depth. Additionally, the synthetic aperture length is limited such that range cell migration is avoided, leading to a depth-dependent Doppler bandwidth and, consequently, resolution. The processing chain is completed by performing clutter suppression, where the four individual SAR images are weighted and coherently combined in a digital signal-processing stage, as indicated in the bottom part of Figure 5.

\section{SURFACE CLUTTER SUPPRESSION}

\subsection{Acquisition geometry}

Surface clutter refers to surface echoes which exhibit the same delay time as the nadir echo from a given depth, i.e. clutter represents an ambiguous return that can mask the desired signal. First, in the simplest case, it is assumed that the effects of topography and airplane roll are absent. In this case, the locus of the clutter on the surface is a circular ring, whose extent is defined by the duration of the transmitted pulse (Fig. 6a). After SAR focusing, the clutter area reduces to a cell whose size is defined by the (projected) across- and along-track resolutions of the SAR, as indicated by the dark gray area in Figure 6a. Azimuth focusing directly provides 


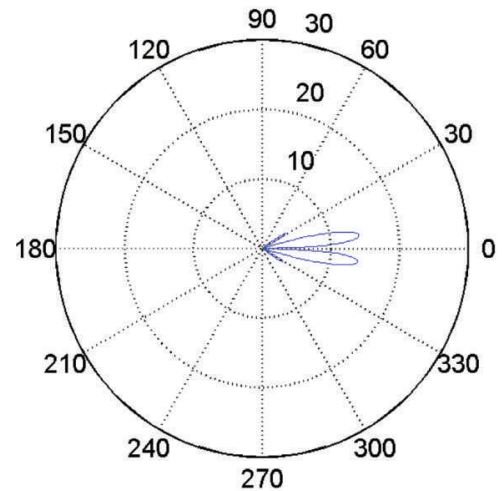

b

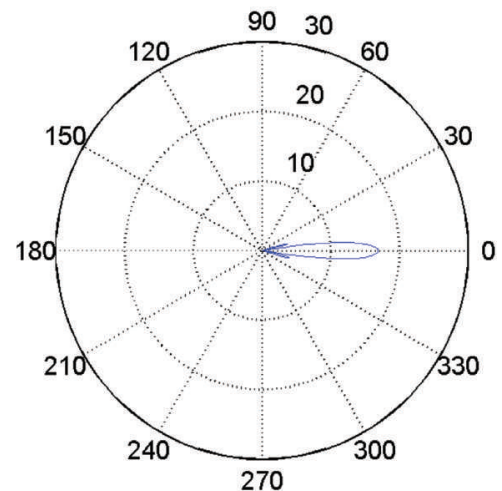

C

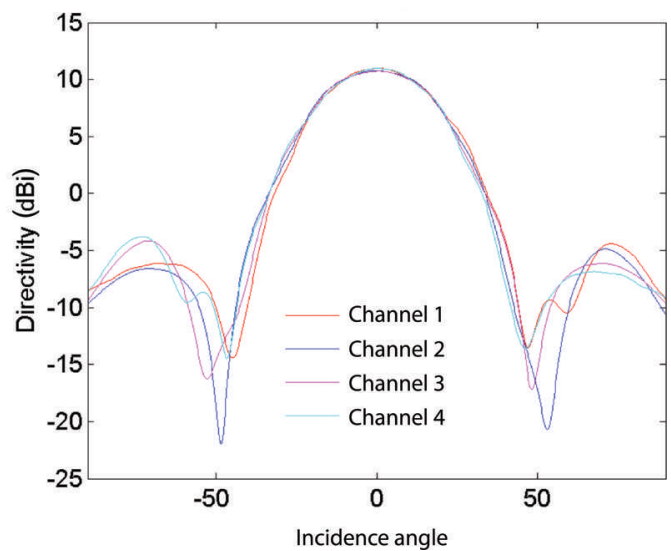

Fig. 3. (a) Transmit antenna pattern of the full aperture, simulated from the measured channel patterns, with the quasi-null at nadir (as operated during the Antarctic multichannel campaign); (b) the measured full aperture on receive; and (c) the measured receive channel patterns.

suppression of the clutter in this dimension, so only two clutter cells in cross-track remain, reducing the clutter suppression approximately to a one-dimensional problem. In a more general approach, topographic variations and an airplane roll angle, $\delta$, are assumed. This can lead to more than two clutter cells, as shown in Figure $6 \mathrm{~b}$, where $\theta_{\mathrm{c}}$ denotes the clutter angles and the nadir angle, $\theta_{\text {nadir, }}$ is the direction of interest.

\subsection{Suppression techniques}

Clutter suppression is based on the coherent weighting and combination of the individually received signals, $j$. The choice of the complex weighting factors, $w_{j}$, for channel $j$ can follow different strategies. In the following, three different techniques are described and individual examples are provided in Figure 7 , where the synthesized receive patterns, $y$, are shown. The pattern, $y$, is simulated by complex weighting, $w_{j}$, and summation of the multiple theoretical (sinc-like) receive channel patterns, $x_{j}$, i.e. $y=w^{\top} x$, with $\mathrm{T}$ the transpose and $x, w$ the vectors of

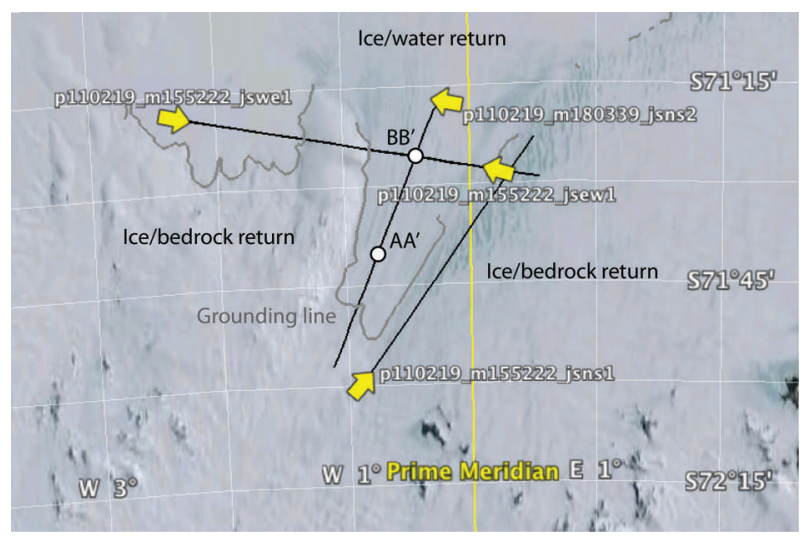

Fig. 4. Google Earth image of the acquired POLARIS multichannel data over Jutulstraumen during the 2011 Antarctica campaign, with arrows indicating the flight direction during acquisition. Dark gray lines show the grounding line around Jutulstraumen from European Remote-sensing Satellite and RADARSAT data, extracted as subset from Rignot and others (2011), indicating the approximate location where grounded ice transitions to a floating mass. Image courtesy of Google Earth, 1 January 1999. elements $x_{j}, w_{j}$, respectively. $y$ is also referred to as the 'joint' antenna pattern in the following. The individual channel patterns, $x_{j}$, were simulated including the phase due to the spacing of the individual POLARIS channels, and thus include the antenna geometry. In the processing as well as the simulation provided below, clutter angles are assumed to be known.

\section{Beam steering (BS)}

In this approach, the weights are chosen such that the antenna pattern is always steered to the angle of interest, i.e. nadir, $\theta_{\text {nadir }}$. Hence, the weight vector, $w$, is defined as

$$
w=s\left(\theta_{\text {nadir }}\right),
$$

where $s\left(\theta_{\text {nadir }}\right)$ is the complex steering vector in the direction of interest (nadir) (Stutzman and Thiele, 1998). This technique maximizes the (thermal) signal-to-noise ratio (SNR) but does not optimize the signal-to-clutter ratio (SCR), where the latter is defined as the ratio between the signal return from the bedrock (nadir) and the clutter reflection

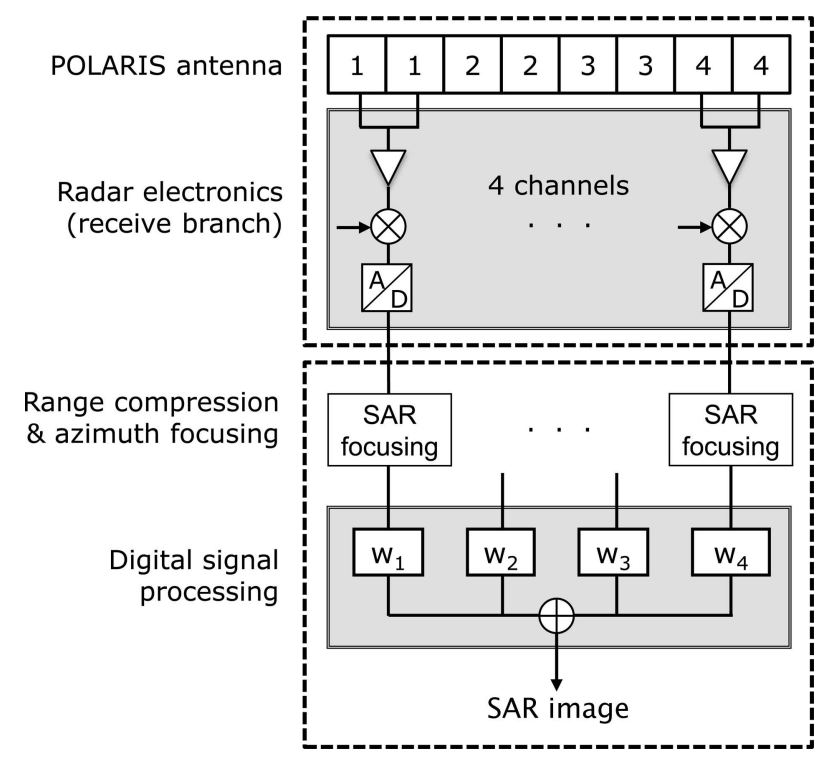

Fig. 5. Instrument (top) and processing chain (bottom) block diagrams. 
a

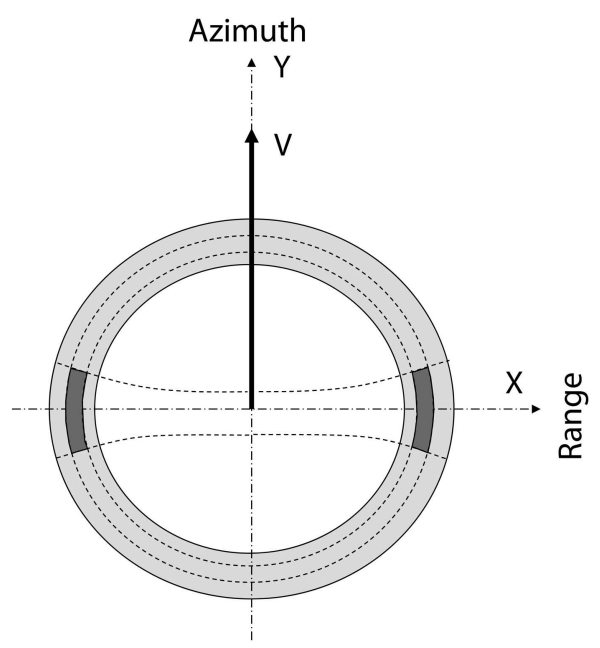

b

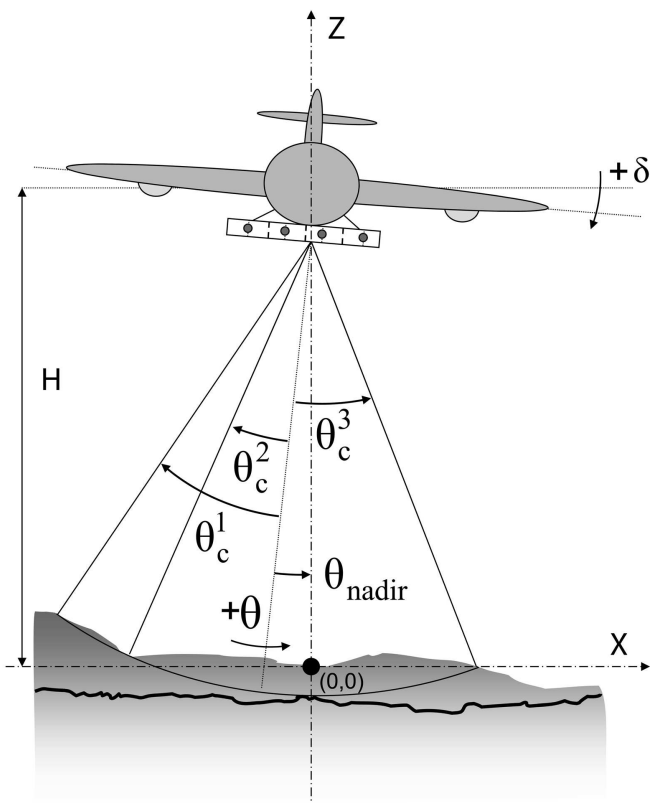

Fig. 6. (a) Surface clutter contribution before SAR processing (light gray locus), and after focusing in azimuth direction by Doppler processing and in range by range compression (dark gray cells). The azimuth or flight direction is indicated by $\mathrm{V}$. This example assumes flat topography in the absence of airplane roll. (b) Acquisition geometry with clutter directions, allowing variation in roll angle and surface topography.

originating from the ice surface at the corresponding travel time. To maintain a constant sensitivity in the nadir ('unitary gain'), a normalization is performed,

$$
w^{\top} s\left(\theta_{\text {nadir }}\right)=1 \text {. }
$$

\section{Null steering (NS)}

Null steering is a method proposed for radar ice sounding by various studies (Velado, 2004; Scheiber and Prats, 2007). It calculates the weights such that nulls are generated in the joint antenna pattern in the expected clutter directions. Ideally, one wants to suppress each clutter direction, i, i.e. $w^{\top} s\left(\theta_{i}\right)=0$, where $s\left(\theta_{i}\right)$ represents the steering vector in the direction of the ith surface clutter cell. For a system of $n$ channels a maximum of $n-1$ nulls can be placed at the same time. Similar to the BS approach, the unitary gain constraint is imposed on the weights, in order to ensure

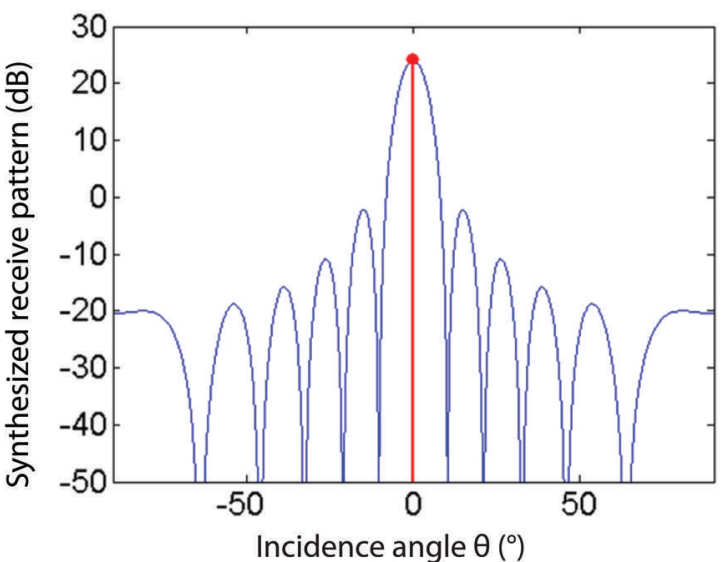

b

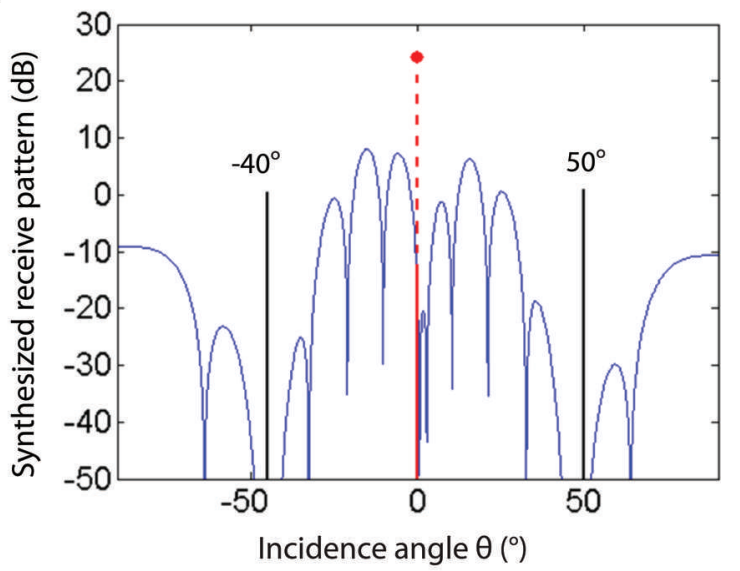

C

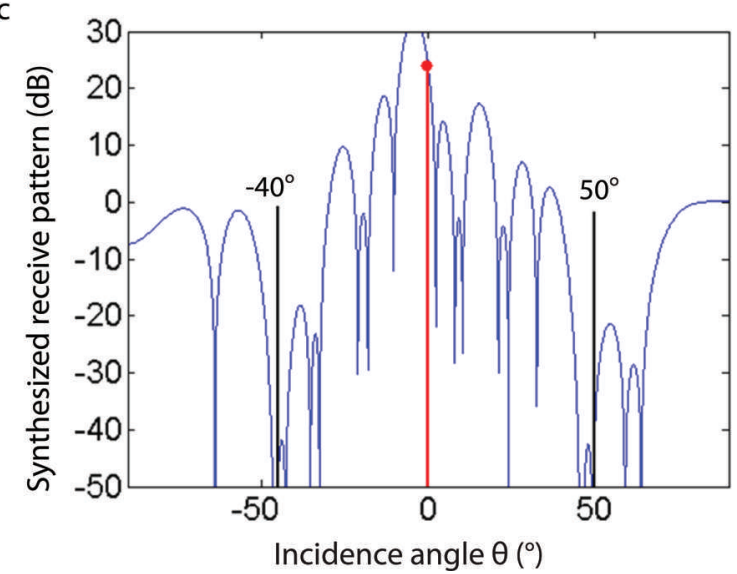

Fig. 7. Synthesized receive antenna pattern from the weighted combination of individual channels in the case of (a) beam steering, (b) null steering and (c) the minimum variance distortionless response. The main beam is steered towards nadir, while the clutter angles are assumed to be at $-40^{\circ}$ and $50^{\circ}$. For the null-steering approach the placing of nulls is at the cost of the nadir gain sensitivity (red marker and dashed red line). Forcing the unitary gain constraint in nadir results in noise scaling.

constant gain in the direction of the nadir:

$$
\left[\begin{array}{c}
s\left(\theta_{\text {nadir }}\right)^{\top} \\
s\left(\theta_{1}\right)^{\top} \\
\vdots \\
s\left(\theta_{i}\right)^{\top}
\end{array}\right] w=\left[\begin{array}{c}
1 \\
0 \\
\vdots \\
0
\end{array}\right] .
$$

This set of equations has infinite solutions when the number of clutter cells is less than $n-1$. To demonstrate the 
technique we do not optimize for the case of infinite solutions, but instead we introduce one or more additional jammers at angles coinciding with nulls in the transmit antenna pattern, so a unique solution is obtained. The NS approach optimizes the SCR but (as it is based on a network of inverse filters) it might scale the noise power and thus degrade the SNR, as detailed by Gebert and others (2009).

\section{Minimum variance distortionless response (MVDR)}

The MVDR, also referred to as the Capon beamformer (Capon, 1969) or optimum beamformer (Guerci, 2003), represents a combination of beam steering and null steering. The weights are determined such that the joint power of noise and clutter is minimized,

$$
\begin{aligned}
& w=Q^{-1} s\left(\theta_{\text {nadir }}\right) \\
& Q=\sigma_{n}^{2} I+\sum_{i=1}^{m} \sigma_{i}^{2} s\left(\theta_{i}\right) s\left(\theta_{i}\right)^{\top},
\end{aligned}
$$

where $Q$ represents the covariance matrix of the colored noise, consisting of the weighted sum of the identity matrix (representing white noise) and the matrices evolving from the steering vectors in the directions of the surface clutter. The power ratio, $\sigma_{i}^{2} / \sigma_{n}^{2}$, is the clutter-to-noise ratio (CNR) for the ith clutter cell. The weights are subject to a unitary gain constraint, as detailed in Eqn (2).

Equation (4) contains both the previous approaches (BS and NS). For CNR $=0$ the MVDR is equivalent to BS, while for $\mathrm{CNR}=\infty$ NS is obtained, making the previous approaches data-independent. In contrast, the MVDR requires a priori knowledge of the CNR or its correct estimation from the data, as investigated in the following subsection.

\subsection{CNR prediction}

The prediction of the CNR simplifies to the prediction of the clutter power, when assuming a constant thermal noise power in the instrument. In theory, the power received from a dedicated clutter cell, $P_{\mathrm{c}, i}^{\text {theory }}$, in the direction of $\theta_{i}$ can be estimated by the well-known radar equation

$$
P_{\mathrm{c}, i}^{\text {theory }}\left(\theta_{i}\right)=\frac{P_{\mathrm{t} x} \lambda^{2} L\left(\theta_{i}\right)}{(4 \pi)^{3} R\left(\theta_{i}\right)^{4}} A\left(\theta_{i}\right) \sigma^{0}\left(\theta_{i}\right) G_{\mathrm{tx} x}\left(\theta_{i}\right) G_{\mathrm{r} x}\left(\theta_{i}\right) \text {, }
$$

with $P_{\mathrm{t} x}$ the transmit power, $G_{\mathrm{t} x}\left(\theta_{i}\right)$ the transmit antenna gain, $G_{r x}\left(\theta_{i}\right)$ the receive antenna gain, $\lambda$ the wavelength, $L\left(\theta_{i}\right)$ containing all additional losses, $R\left(\theta_{i}\right)$ the slant range distance, $\sigma^{0}\left(\theta_{i}\right)$ the backscatter coefficient and $A\left(\theta_{i}\right)$ the instantaneously illuminated area on the ground. After SAR focusing, $A\left(\theta_{i}\right)$ is substituted by the number of compressed samples in azimuth, $N_{\mathrm{az}}$, and range, $N_{\mathrm{rg}}$, and the respective ground (surface) resolutions, $\delta_{\mathrm{az}}$ and $\delta_{\mathrm{rg}}\left(\theta_{i}\right)$. The latter evolves from the projection of the slant range resolution, $\delta_{\mathrm{s}}$, on the ground and depends on the incidence angle, $\theta$ :

$$
\begin{aligned}
A\left(\theta_{i}\right) & \approx N_{\mathrm{az}} \delta_{\mathrm{az}} \cdot N_{\mathrm{rg}} \delta_{\mathrm{rg}}\left(\theta_{i}\right) \\
\delta_{\mathrm{rg}}\left(\theta_{i}\right) & =\sqrt{\left(\frac{H}{\cos \theta_{i}}+\delta_{\mathrm{s}}\right)^{2}-H^{2}}-\sqrt{\left(\frac{H}{\cos \theta_{i}}\right)^{2}-H^{2}},
\end{aligned}
$$

where $H$ is the altitude above the surface. Equations (5) and (6) then allow prediction of the clutter power. One observes that the clutter power, and thus the CNR, varies with incidence angle. It can be split into a constant weighting, $\mathrm{CNR}_{0}$, relating clutter and noise at a reference angle, $\theta_{0}$ (e.g.
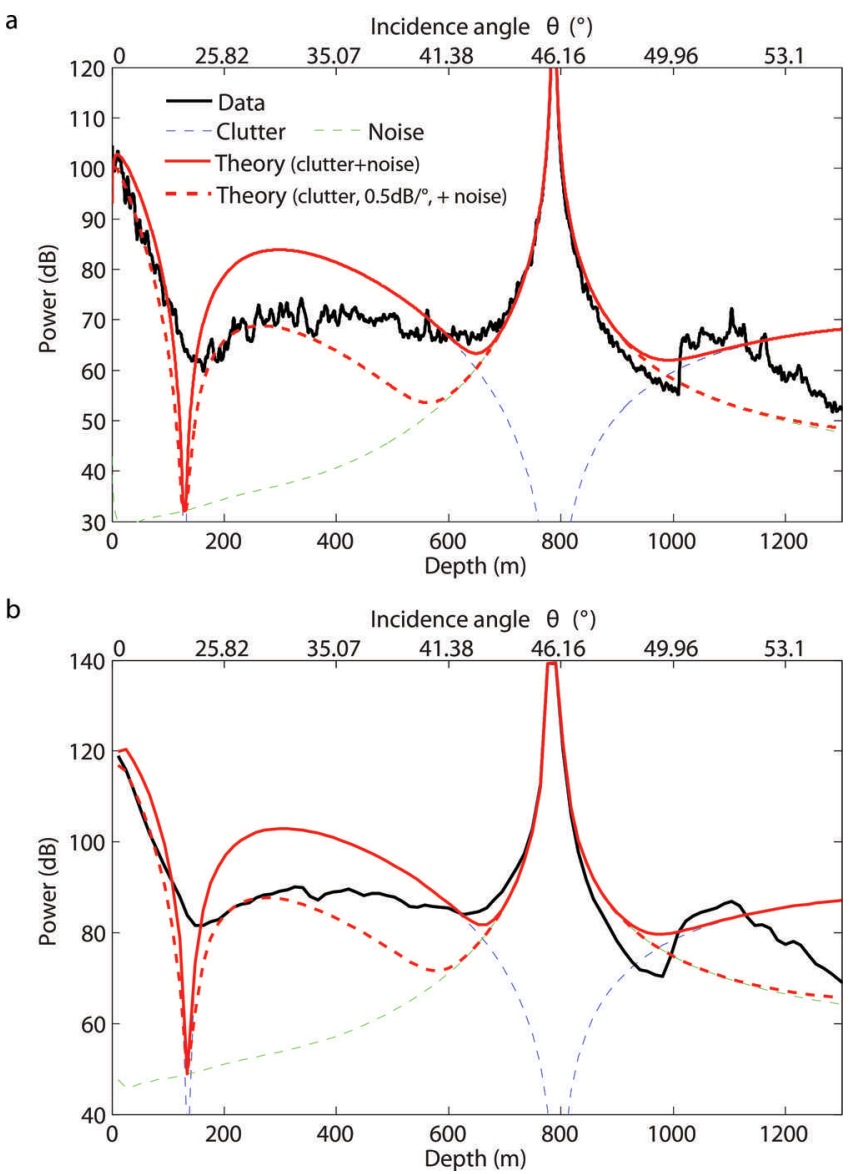

Fig. 8. Jsns 2 track at $50 \mathrm{~km}\left(\mathrm{BB}^{\prime}\right.$ in Fig. 4) along-track distance average over a $200 \mathrm{~m}$ azimuth range. (a) Measured clutter signal (black) with the $85 \mathrm{MHz}$ bandwidth compared to its prediction (red), being the sum of scaled noise (green) and estimated clutter (blue). In addition, a theoretical estimate is provided, assuming a linear backscatter decrease of $0.5 \mathrm{~dB}$ per degree (dashed red), showing an improved correlation with the data. Strong noise scaling occurs at the grating lobe equivalent to $\sim 800 \mathrm{~m}$ depth. The ice bottom return corresponds to a depth of $1000 \mathrm{~m}$. (b) Same as (a) but for $6 \mathrm{MHz}$ bandwidth.

the nadir), and a normalized variation of the clutter power.

$$
\operatorname{CNR}(\theta)=\mathrm{CNR}_{0}\left(\theta_{0}\right) \frac{P_{\mathrm{C}}^{\text {theory }}(\theta)}{P_{\mathrm{C}}^{\text {theory }}\left(\theta_{0}\right)} .
$$

The angular variation of the clutter power is dominated by the joint antenna patterns and $\sigma^{0}(\theta)$. While the patterns are accurately known, $\sigma^{0}(\theta)$ is subject to uncertainties as it depends strongly on angle, frequency and ice surface characteristics, and thus limits the accuracy of the prediction.

In order to validate the above model approach we made a comparison between the surface clutter power estimated from the data, $\mathrm{AA}^{\prime}$ profile of the Jsns 2 track data at $50 \mathrm{~km}$ along-track distance, and the theoretical prediction. Low roll variation and small across-track topography variations at $50 \mathrm{~km}$ along-track distance allow us to simplify the surface clutter problem to clutter cells positioned symmetrically about the nadir. The signal power, $P_{\mathrm{NS} \text {, nadir }}(\theta)$, as shown in Figure 8 (black), is computed by complex weighting of each channel, for which the weights follow from NS towards nadir, while maintaining a unitary gain towards the surface reflection, $\theta_{\mathrm{c}}$. The signal power curve (black) is obtained by averaging the range power over a $200 \mathrm{~m}$ along-track 
Table 2. Parameters for sensitivity analysis

\begin{tabular}{lc} 
Parameter $(50 \mathrm{~km}$ along track) & Value \\
\hline Roll angle & $0^{\circ}$ \\
Altitude above ground & $3244 \mathrm{~m}$ \\
Index of refractivity & 1.8 \\
Topography & flat \\
Transmitted bandwidth & $\infty / 85 \mathrm{MHz}$ \\
Antenna patterns & theory \\
\hline
\end{tabular}

distance. The approach suggested here is the reverse of Eqn (3), which is written for a NS towards the surface clutter cell directions with a normalization constraint at nadir. As the clutter cells have identical angles of opposite signs, the normalization constraint works in either of the two clutter directions. Due to the inverse character of the NS, the obtained signal, $P_{\mathrm{NS} \text {, nadir }}(\theta)$, is a superposition of the surface clutter power, $P_{\mathrm{c}}(\theta)$, the scaled noise power, $P_{\mathrm{n}}|\kappa(\theta)|^{2}$, and scaled off-nadir subsurface signals, referred to as volumetric clutter, $P_{\text {vol }}(\theta)$, leading to

$$
P_{\mathrm{NS}, \text { nadir }}(\theta)=P_{\mathrm{c}}(\theta)+P_{\mathrm{n}}|\kappa(\theta)|^{2}+P_{\mathrm{vol}}(\theta) .
$$

The noise amplitude scaling factor, $\kappa(\theta)$, follows directly from the normalized weights, computed during NS processing, as

$$
\kappa(\theta)=\frac{w^{\top} s(\theta)}{w^{\top} s\left(\theta_{\mathrm{C}}\right)}=w^{\top} s(\theta),
$$

with $w$ the vector containing the complex channel weights, and $s(\theta)$ and $s\left(\theta_{\mathrm{c}}\right)$, respectively, the complex steering vector in the direction of $\theta$ and one of the surface clutter cell directions, $\theta_{\mathrm{c}}$.

Off-nadir volume scattering cannot be avoided. Any large ice inclusions and inhomogeneity would mask the nadir echo, but such situations are not frequent. The internal layering mostly produces specular reflections, thus not visible in off-nadir directions. In most cases, surface and bedrock reflections will be stronger than the volume scattering (Dowdeswell and Evans, 2004). Hence, neglecting the volume contributions from off-nadir directions (while keeping in mind that they might be one of the reasons for some residual offsets between simulations and data) appears to be a valid approach, as demonstrated by the results in this paper. In our theoretical prediction, $P_{\mathrm{NS} \text {, nadir, }}^{\text {theory }}$ we account for the clutter and noise power variations, but due to a limited knowledge of internal scattering we neglect the volumetric contributions, leading to

$$
P_{\mathrm{NS}, \text { nadir }}^{\text {theory }}=c_{1} P_{\mathrm{c}}^{\text {theory }}(\theta)+c_{2}|\kappa(\theta)|^{2},
$$

with $c_{1}$ and $c_{2}$ constants constraining the absolute reference levels of the total theoretical clutter power, $P_{\mathrm{C}}^{\text {theory }}(\theta)$, and the noise scaling, $|\kappa(\theta)|^{2}$, where the noise scaling follows from the weights used when computing a NS towards nadir. For the latter we used the weights as obtained from NS processing of the measured data. The total clutter estimate from the data is a combination of both clutter cells and a superposition of all scatterers within the ground resolution cell. We estimate this by combining both the theoretical clutter power for a dedicated angle, Eqn (5), and by integrating the clutter contribution over the resolution cell, resulting in an average weighted gain. We modeled the transmit antenna gain with a null in nadir and the individual channel gains by using theoretical sinc patterns approximating the real POLARIS antenna. Having estimated the relative variations of clutter and noise power at hand, the last step consists of estimating their absolute reference values by modifying $c_{1}$ and $c_{2}$. This is done manually by matching the relative prediction with the data estimate at two dedicated depths, which are chosen such that either clutter or noise power is dominant. Specifically, the theoretical prediction is aligned with the data at the grating lobe ( $\sim 46^{\circ}$, or $\sim 800 \mathrm{~m}$ depth) and at an angle just off-nadir. At the grating lobe the clutter power is nulled, so the only remaining contribution comes from the noise scaling. In contrast, the off-nadir angle is dominated by clutter. As additional validation, we found our noise power estimate to align well with the signal power received before the nadir surface return.

After this the theoretical prediction, $P_{\mathrm{NS} \text {, nadir }}^{\text {theory }}(\theta)$ (Fig. 8 (red) and Eqn (10)), follows from the sum of the scaled noise power, $c_{2}|\kappa(\theta)|^{2}$ (dashed green), and the total theoretical clutter, $c_{1} P_{c}^{\text {theory }}(\theta)$ (dashed blue). Backscatter has not been modeled. In contrast, the dashed red curve applies a simple model assuming a constant drop of $0.5 \mathrm{~dB}$ per degree incidence angle for the backscatter, leading to a good correlation between the prediction (dashed red) and the estimate from the data (black). Although the clutter is underestimated in some regions, one can observe a good match of the trends, in particular for depths of strong clutter up to $\sim 500 \mathrm{~m}$. For depths below $1000 \mathrm{~m}$, the corresponding incidence angles are $50^{\circ}$ and larger, meaning that the simple model for a constantly dropping backscatter coefficient with angle is no longer applicable.

\subsection{Sensitivity analysis}

The sensitivity analysis presented in this subsection compares the different techniques introduced in Section 2.2. Specifically, the impact of the applied technique on noise power and on clutter power is investigated. The results are obtained by evaluating the weighting functions of each technique for the relevant range of incidence (clutter) angles and the corresponding ice depth. All simulations are performed using theoretical sinc patterns approximating the POLARIS set-up and assuming steady flight over flat terrain, as summarized in Table 2. This compares well with the test dataset of the next section. For the minimum variance distortionless response approach a $\mathrm{CNR}_{0}$ value of $60 \mathrm{~dB}$ is applied. As shown below, we found this $\mathrm{CNR}_{0}$ value to approximate reasonably well the optimum achieved clutter suppression.

The 'noise power scaling' is defined as the increase of thermal noise power with respect to the reference, as it follows from Eqn (9). The scaling of the noise is solely based on the normalization of the weights and thus is bandwidthindependent. Strictly speaking, when translating the scaled noise level to a change of SNR, the variation of signal power across the resolution cell needs to be accounted for. However, the impact of the two-way average gain within the ground resolution cell for the signal (nadir) is small and its variation is neglected here. Hence, the variation of noise power directly represents the change of SNR.

Figure 9a shows the results for the different techniques. As expected, the beam steering (BS, red curve) gives the 
optimum solution in providing a constant SNR for any ice depth, but the minimum variance distortionless response approach (MVDR, blue curve) performs only slightly worse. The worst result is obtained by null steering (NS, green curve), which exhibits a stronger scaling than the MVDR and tends to infinity around the grating lobe at $\sim 46^{\circ}$. Note that this is not a realistic case, as thermal noise is essentially neglected; however, it was chosen to demonstrate the strong noise scaling caused by the inverse character of the weighting functions which leads to a strong degradation of SNR, in particular in the vicinity of the grating lobe.

The 'Clutter-suppression performance' is defined as the strength of the return integrated over the surface resolution cell. Hence, in contrast to the noise, the clutter power is bandwidth-dependent. Again, the signal power is assumed to be constant, independent of the resolution cell size.

As a reference, Figure $9 \mathrm{~b}$ gives the clutter level assuming an infinite bandwidth. In this case the ground resolution cell becomes so small that no averaging is required. Beam steering (red) exhibits the poorest performance in terms of clutter suppression, as the approach is optimized for the SNR only; the clutter power simply follows the shape of the antenna pattern. Null steering (green) provides perfect suppression of the clutter as it places a true null in the direction of the clutter cell. However, this comes at the cost of a degraded thermal SNR (Section 2.2). Finally, MVDR (blue) shows particularly good suppression of the strong echoes at low depths at the cost of a moderate noise increase. When getting close to the grating lobe, the MVDR follows closely the BS characteristic, thereby avoiding any strong noise scaling.

As a second case, results for a transmit bandwidth of $85 \mathrm{MHz}$ are presented in Figure 9c. Compared to the result of Figure 9b, one observes a clearly degraded performance for NS (dashed green) over the full depth range. This is caused by the fact that the placed null is much narrower than the resolution cell size over which the integration is performed. This is also the reason for the performance loss of the MVDR, which is much less distinct than that for NS. The effect on BS is limited and overall corresponds to a low-pass filtering of the antenna pattern. The impact of the transmitted bandwidth is expected to be more severe the coarser the resolution becomes.

This leads to the important conclusion that the original optimum weights used might not represent the optimum solution once the resolution cell size has been taken into account. A deep but narrow null might completely suppress the clutter from this specific direction, but at the same time neglect the clutter from other directions within the same resolution cell. As a consequence, possible optimization strategies could aim to develop weighting factors that provide reasonable suppression over a broader area. This could be achieved by placing two dedicated nulls close to each other within the same resolution cell, but in the present case (where only a maximum of three nulls can be generated) such a 'workaround' is not applicable. In general, the larger the transmitted bandwidth, the smaller the resolution cell and thus the better the narrow null removes clutter from the complete resolution cell. This means that an increased bandwidth is beneficial for the positioning of the nulls. Unfortunately, there is also a drawback because mis-registration between channels becomes more severe for larger bandwidths. Mis-registration means that different channels start to see the same clutter a

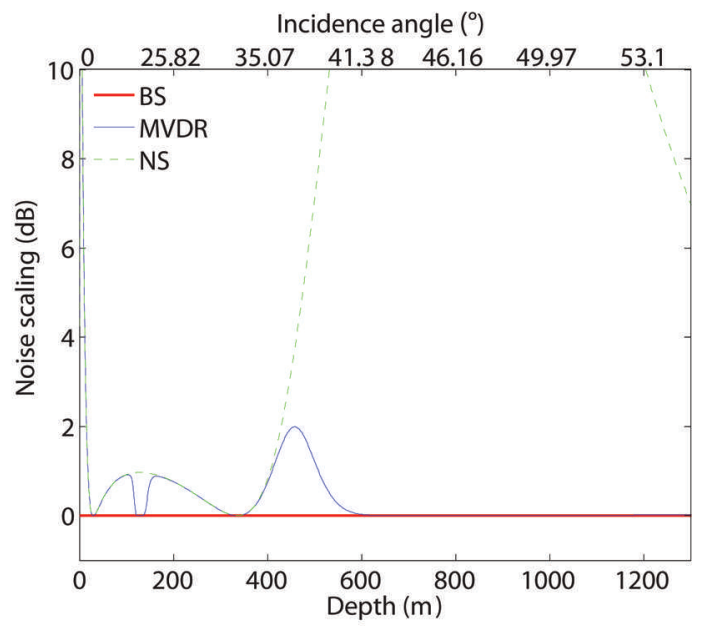

b

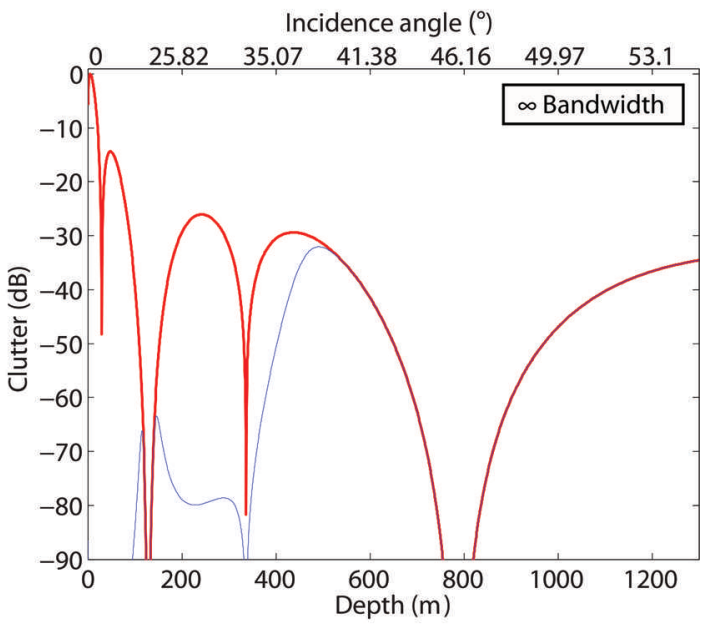

c

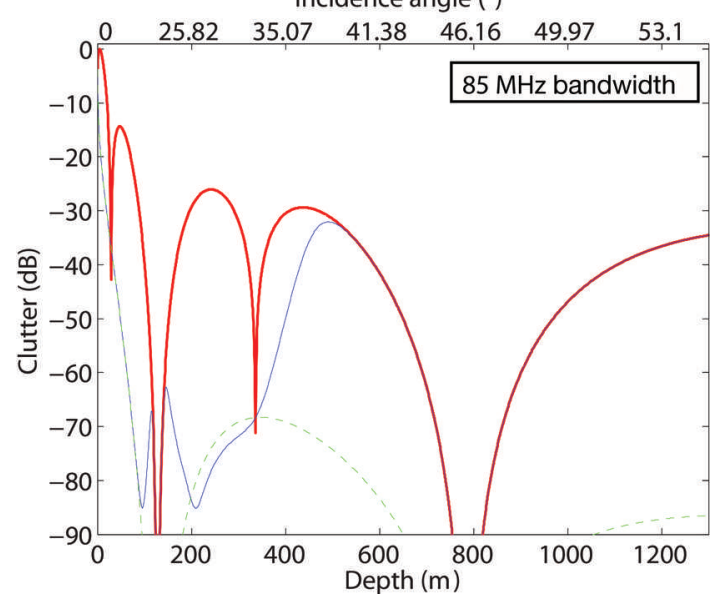

Fig. 9. Sensitivity analysis of (a) SNR degradation due to noise scaling and (b, c) clutter-suppression capability for (b) an infinite bandwidth and (c) a bandwidth of $85 \mathrm{MHz}$. BS (red), NS (dashed green) and the $M V D R$ for $\mathrm{CNR}_{0}=60 \mathrm{~dB}$ (blue) show the behavior of the different processing methods. A performance drop is observed for a smaller bandwidth, due to integration over the ground resolution cell. Assumed parameters are summarized in Table 2.

from a specific angle at different times, i.e. potentially in different range lines of the stored data. While for the $6 \mathrm{MHz}$ bandwidth this is not a problem, it does introduce a phase error between channels for $85 \mathrm{MHz}$ bandwidth and a performance drop can be expected for incidence angles $>10^{\circ}$ (Nannini and others, 2013). 
Closely linked to the above discussion is another important issue. As mentioned above, the weights are normalized to unitary gain at nadir, which means that only one dedicated direction is used to define the desired signal. This neglects the returns from off-nadir subsurface reflections, and their potential weighting by the synthesized pattern resulting from the weighting coefficients. As a result, identical signal power can no longer be expected for BS and MVDR, and integration of the weighted subsurface returns would be required.

\section{APPLICATION TO MEASURED DATA}

Here we present the multichannel processing results applied to the Jsns2 and Jsew1 tracks covering Jutulstraumen (Fig. 4). In total, four channels are received, digitized and stored, and then combined using the different clutter-suppression techniques described above. Processing is performed by a tool developed at ESA (Villano, 2009; Bekaert, 2012), which calculates the weights depending on the geometry (topography, airplane roll, etc.) and the system parameters (antenna patterns, etc.). This includes estimation of the CNR for a specific angle only, Eqn (10). In our processing, channel patterns have been approximated by theoretical sinc patterns, and thus do not include channel imbalances. While currently not implemented, expanding the processor with the measured patterns would allow phase imbalances to be accounted for, which is expected to improve the clutter-suppression performance. Clutter angles are computed assuming a flat surface. This approximation appears reasonable, as the data were acquired over a horizontal ice shelf in hydrostatic equilibrium. Investigations for external digital elevation model (DEM) data were performed regarding the $500 \mathrm{~m}$ resolution Ice, Cloud and land Elevation Satellite (ICESat)/Geoscience Laser Altimeter System (GLAS) DEM and the $30 \mathrm{~m}$ resolution Advanced Spaceborne Thermal Emission and Reflection Radiometer (ASTER) GDEM2. However, due to large residuals between the DEMs and the estimated ice surface from POLARIS, likely introduced due to the lower spatial resolution and different acquisition time, both DEMs were disregarded. In the future, inclusion of topographic data - using an external DEM or direct estimation of the surface return from the data itself (Paden and others 2010; Wu and others, 2011; Nielsen and others, 2012; Li and others, 2013) by various direction-of-arrival estimation methods (Schmidt, 1986; Stoica and Nehorai, 1989; Jezek and others, 2011) including beam steering, MUSIC (MUltiple SIgnal Classification), maximum-likelihood and radar interferometry - represents a means of improving the clutter-suppression result.

\subsection{Full track analysis}

First, the clutter-suppression results for the complete flight tracks Jsns2 and Jsew1 are presented in Figures 10 and 11, respectively. For Jsns2 both the 6 and $85 \mathrm{MHz}$ bandwidths are shown. They have different SNRs as they were acquired with different pulse durations. For Jsew1, only the $85 \mathrm{MHz}$ bandwidth is considered.

In Figures 10 and 11 the top row represents the signal as received by a single channel (along-track focused), i.e. no multichannel clutter suppression is applied. The clutter signal is clearly present and appears as a smooth signal that covers any feature in the ice up to a depth of several hundred meters. It starts becoming less significant only at a depth of 500-600 m. At the start of the Jsns2 track (Fig. 10) the strong surface reflection extends up to depths of $300-400 \mathrm{~m}$. This feature coincides with large roll variations of up to $20^{\circ}$ at the start of the track (Fig. 12). The high-power subsurface signals correlate spatially with the segments of floating ice, and thus represent the reflection at the ice/ water boundary. We find the transition from the high- to lower-power return to correlate with the transition from a floating ice mass to a grounded ice stream. This correlates with the location of the grounding line (Rignot and others, 2011), shown in Figure 4. In the case of Jsew 1 (Fig. 11) the bedrock return is masked by surface clutter. While the clutter is expected to be stronger than the nadir signal, this feature is enhanced by the null on transmit, decreasing the antenna sensitivity at nadir.

The second row shows the clutter-suppressed result, obtained by regular beam steering (BS) of the signal towards nadir. There is a clear improvement, as can be seen from the strongly reduced clutter power in the region from the surface to $500-600 \mathrm{~m}$. In particular, the contrast of the ice bottom becomes much stronger and sharper. The bedrock reflection for Jsew 1 has now become more clearly visible.

The third row gives the result after applying null steering (NS), which shows clearly improved clutter suppression in the depth range $200-320 \mathrm{~m}$, as highlighted by the black rectangular boxes. Note that two nulls were placed in the direction of the two clutter cells. Rather than using the remaining degree of freedom for optimizing the weights, for simplicity we introduced an additional null at $22^{\circ}$, being a natural null in the transmit antenna pattern (Fig. 3), such that a unique solution was obtained at no additional cost. One can observe the smearing of the reflection signals, caused by signal scaling introduced by the unitary gain constraint. At the grating lobe the inverse character of the noise scaling (unitary gain constraint at nadir) results in a strong power increase. The location where the noise scaling appears as two signals corresponds to the locations at which roll variations result in an asymmetric clutter geometry and consequently two different depths are associated with the left and right grating lobe. Note that the color bar has been saturated, allowing a comparison with the other multichannel processing techniques.

Finally, the bottom row gives the optimum result obtained with the minimum variance distortionless response (MVDR) by varying the $\mathrm{CNR}_{0}$. For each depth we evaluated and retained the solution that had the largest reduction in signal power. In other words, any reduction of power level is considered as a suppression of clutter. This is an approximation, as in reality one should keep in mind that a potential scaling of off-nadir subsurface returns ('volume clutter') is neglected, as mentioned above. We found that a nonconstant $\mathrm{CNR}_{0}$ yields the optimum result. This leads to the conclusion that the model describing the clutter (Eqn (7)) is not perfect. However, the variations of the optimum $\mathrm{CNR}_{0}$ versus depth give a measure of the residual errors in the model. As the variations were not significant we assume the model to be applicable. A comparison with a single $\mathrm{CNR}_{0}$ is made below. Compared to BS, further reduction of clutter power is observed within the depth range 200-320 m, as highlighted by the black rectangular boxes. Compared to the NS, big improvements can be observed in the vicinity of the grating lobes because the MVDR avoids any excessive scaling of noise. As expected, the echo from the ice bottom return remains clearly visible. 

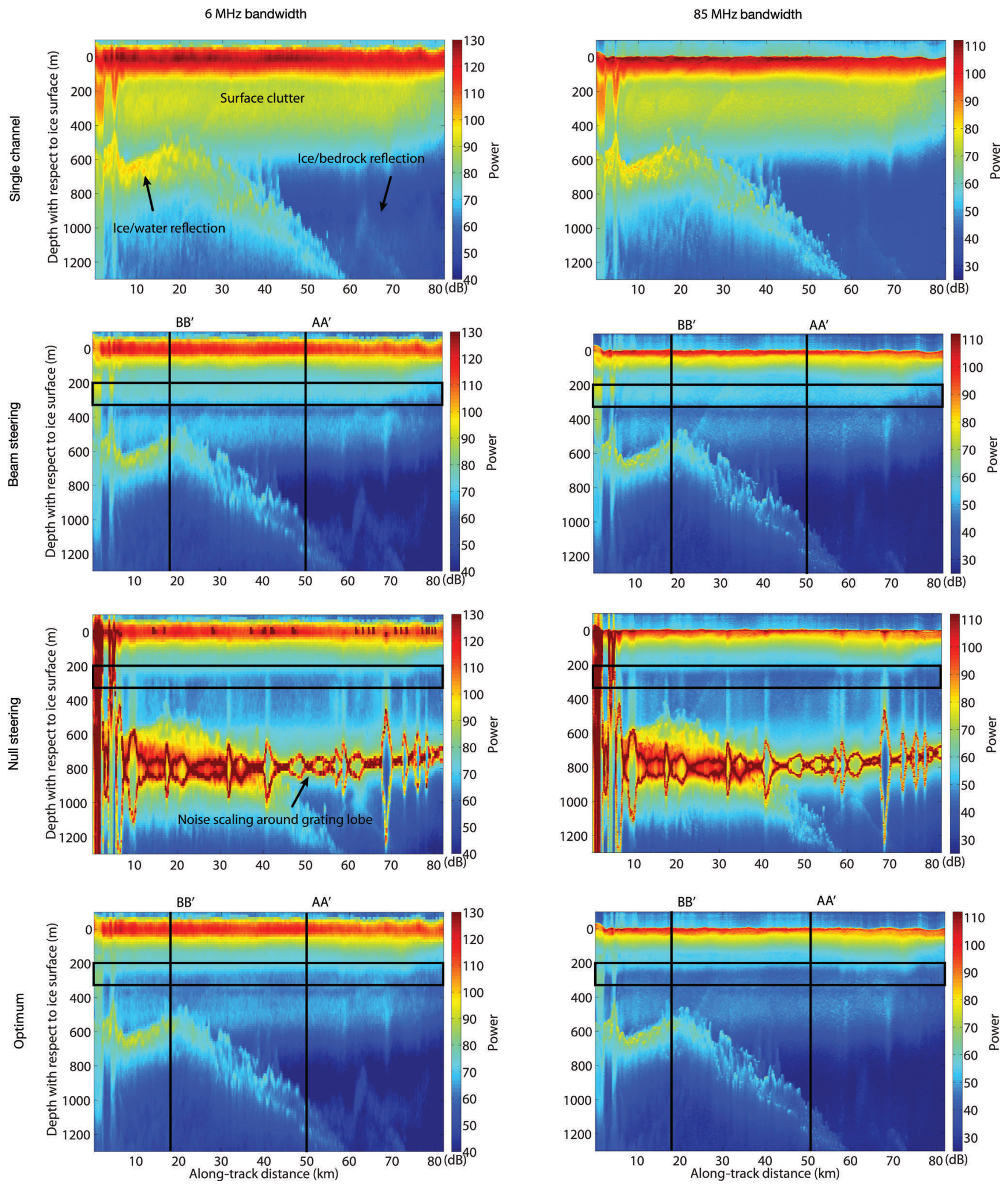

Fig. 10. Glacier center-line upstream flight track (Jsns2). The rows from top to bottom show the sounding result from a single receive channel after azimuth processing, the result after regular BS, the result after NS, and the optimum obtained after applying the MVDR for a varying set of $\mathrm{CNR}_{0}$. The columns give the sounding results for $6 \mathrm{MHz}$ (left) and $85 \mathrm{MHz}$ (right) bandwidths. For the NS, strong noise scaling occurs at and around the grating lobe depth at $\sim 800 \mathrm{~m}$. The highlighting in the black boxes clearly shows reduced clutter contributions at depths of $200-500 \mathrm{~m}$. The bedrock starting at $600 \mathrm{~m}$ depth and reaching $>1200 \mathrm{~m}$ is clearly visible and marked by the red dashed line. $\mathrm{AA}^{\prime}$ and $\mathrm{BB}^{\prime}$ are the power profiles shown in Figures 13 and 14, respectively.

\subsection{Data cross sections}

In this section, BS and the MVDR are compared in more detail by means of a quantitative analysis of the intensity profile cuts, which are taken at the along-track positions, lines $\mathrm{AA}^{\prime}$ and $\mathrm{BB}^{\prime}$ (Fig. 4). The NS is no longer included at this stage. We include a comparison for a fixed $\mathrm{CNR}_{0}$ value of $60 \mathrm{~dB}$, estimated by comparing the received signal peak just offnadir with the noise level after receiving the bedrock signal. 

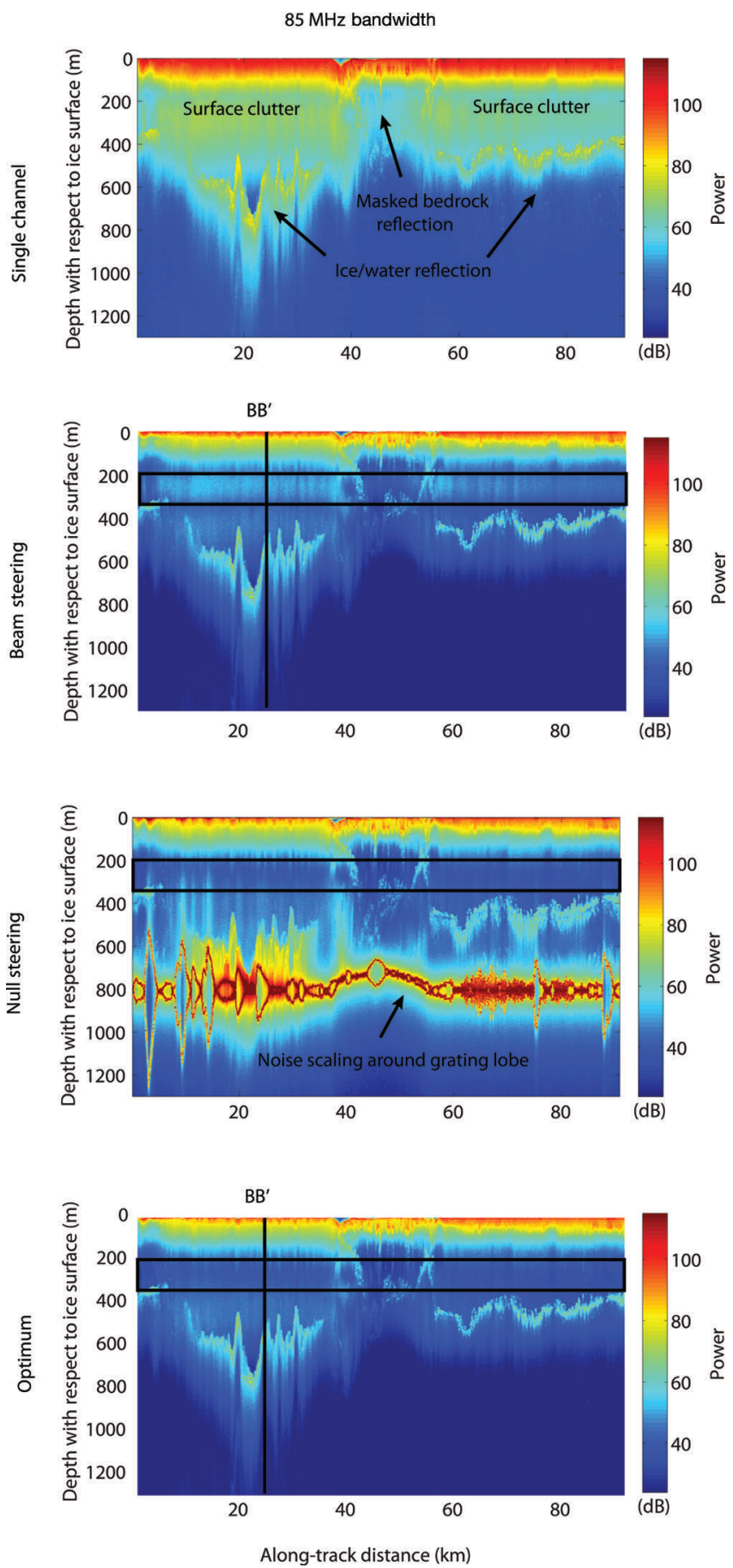

Fig. 11. Flight track perpendicular to the glacier (Jsew1). From top to bottom the $85 \mathrm{MHz}$ bandwidth sounding result from a single receive channel after azimuth processing, the result after regular $\mathrm{BS}$, the result after NS, and the optimum obtained after applying the MVDR for a varying set of $\mathrm{CNR}_{0}$. For the NS, strong noise scaling occurs at and around the grating lobe depth at $\sim 800 \mathrm{~m}$. The black boxes highlight the clear reduction in clutter contributions at depths of $200-500 \mathrm{~m}$. The bedrock, starting at $600 \mathrm{~m}$ depth and reaching $>1200 \mathrm{~m}$ is clearly visible and marked by the red dashed line. BB' corresponds to the power profile shown in Figure 14.

\section{The $A A^{\prime}$ profile}

The $\mathrm{AA}^{\prime}$ profile is presented in Figure 13 for both the 6 and $85 \mathrm{MHz}$ bandwidths of the Jsns2 track. With respect to the $6 \mathrm{MHz}$ bandwidth, the $85 \mathrm{MHz}$ bandwidth dataset appears to be shifted $\sim 18-19 \mathrm{~dB}$, corresponding well with the calibration factor of $18 \mathrm{~dB}$, that is not included. The
Jsns2
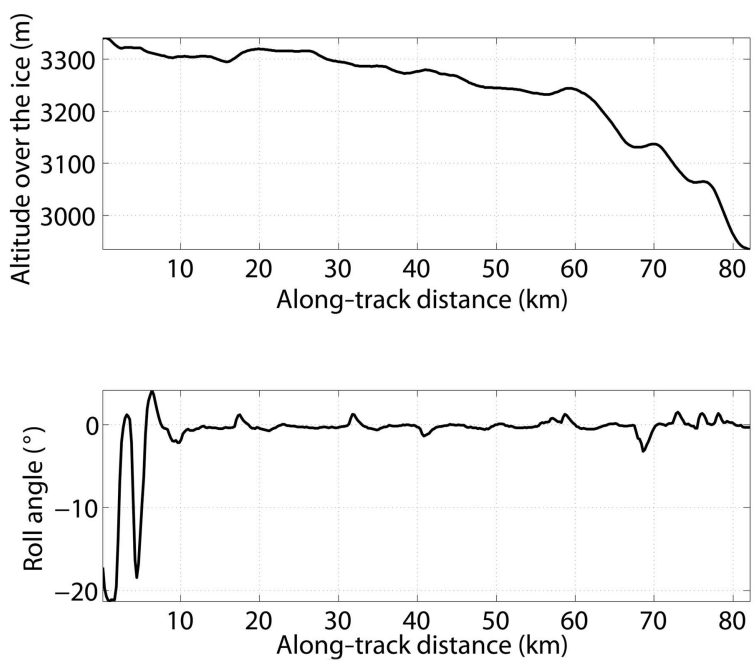

Jsew1
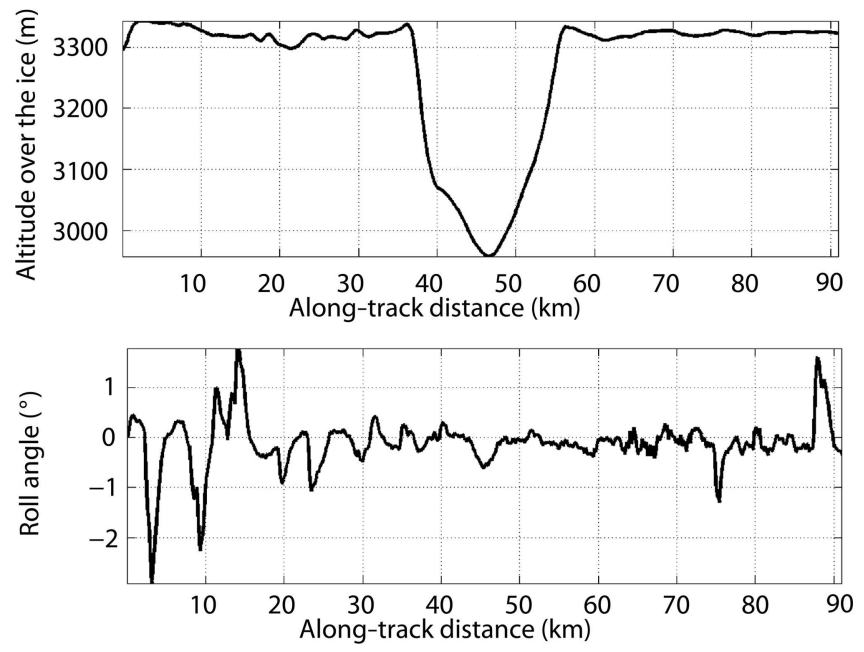

Fig. 12. Variation of altitude and roll angle along-track for the Jsns2 track (above) and the Jsew1 track (below).

reference (black curve), corresponding to BS, is overlaid with the results of the MVDR for a fixed $\mathrm{CNR}_{0}$ value of $60 \mathrm{~dB}$ (blue dashed curve), and a variable $\mathrm{CNR}_{0}$, where different $\mathrm{CNR}_{0}$ are used to generate the weights and then the result of maximum signal reduction is kept (red curve). Note that this high $\mathrm{CNR}_{0}$ value is different from NS, as near the grating lobe the MVDR will tend towards BS and thus will not exhibit the strong noise-scaling behavior.

Comparing the different suppression results, the strongest clutter suppression, of nearly $10 \mathrm{~dB}$, is observed in the region from 200 to $320 \mathrm{~m}$ depth, for which a magnified view is provided in Figure 13b. This is obtained nearly identically for a $\mathrm{CNR}_{0}$ of $60 \mathrm{~dB}$ (dashed blue curve) and the variable $\mathrm{CNR}_{0}$ (red curve), meaning that a $\mathrm{CNR}_{0}$ of $60 \mathrm{~dB}$ represents the optimum of this depth range. However, in the region $\sim 350 \mathrm{~m}$, this value does not yield the optimum solution, but is slightly worse than BS (cf. dashed blue and black curves), while a different $\mathrm{CNR}_{0}$ now gives the optimum solution (red). Below $400 \mathrm{~m}$ depth, again an improvement of several $\mathrm{dB}$ compared with $\mathrm{BS}$ is achieved for both MVDR curves. Figure 13c zooms in to the intensity profile up to $150 \mathrm{~m}$ depth, revealing a similar trend in optimum suppression for both the 6 and 

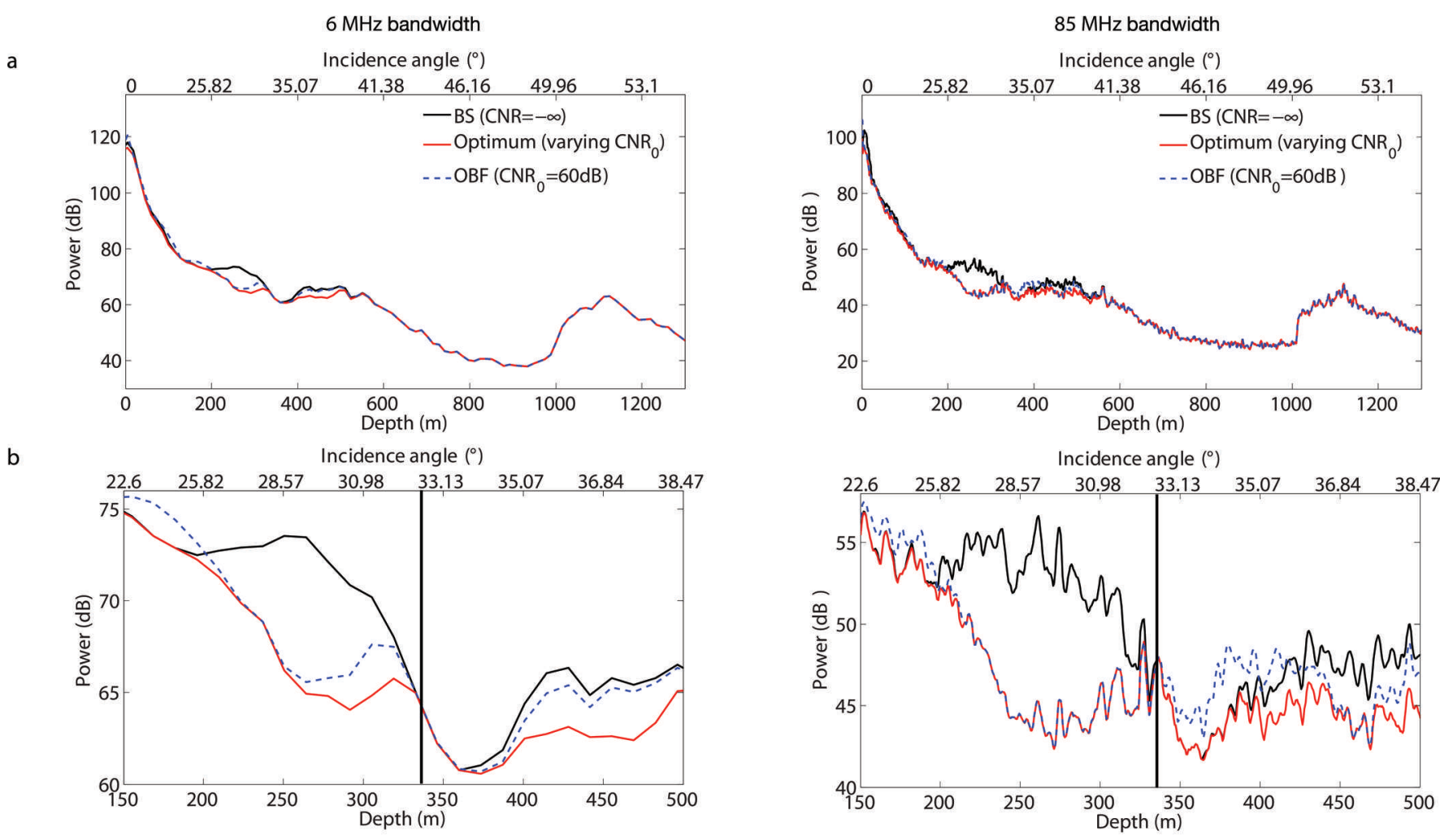

C
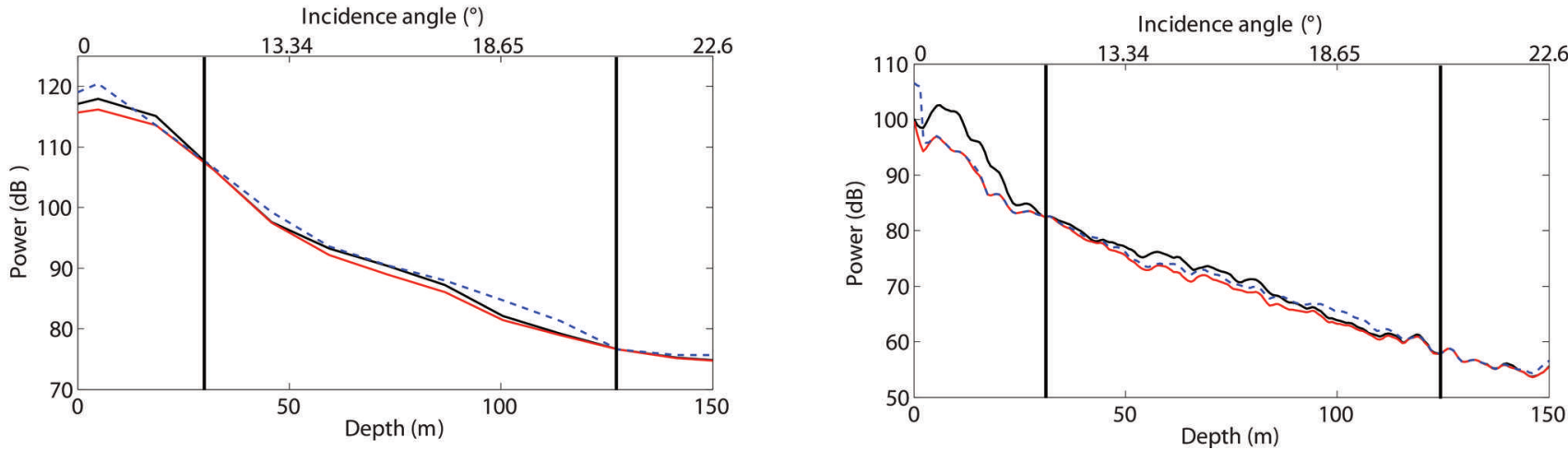

Fig. 13. (a) $A A^{\prime}$ power profile versus depth made at $50 \mathrm{~km}$ along-track distance. BS (black curve) compared with MVDR (dashed blue curve) using a $\mathrm{CNR}_{0}$ of $60 \mathrm{~dB}$ for both the 6 and $85 \mathrm{MHz}$ bandwidths. (b) Zoom from 150 to $500 \mathrm{~m}$. (c) Zoom on the first $150 \mathrm{~m}$. The optimum suppression result (red curve) in terms of combined minimization of clutter power and scaled volumetric scattering and noise is obtained by sampling a variety of $\mathrm{CNR}_{0}$. Suppression up to $5 \mathrm{~dB}$ can be observed for $0-200 \mathrm{~m}$ depths and even up to $10 \mathrm{~dB}$ for depths between 200 and $325 \mathrm{~m}$. The 6 and $85 \mathrm{MHz}$ bandwidths show similar trends in clutter-suppression performance. The bedrock is observed at $\sim 1000 \mathrm{~m}$ depth. Vertical lines indicate the places with natural nulls in the transmit pattern or where the weights generate a null in the clutter direction, resulting in similar suppression performance for all techniques.

$85 \mathrm{MHz}$ bandwidths. However, for the $85 \mathrm{MHz}$ bandwidth the $\mathrm{CNR}_{0}$ approaches the optimum much better, having clutter suppression up to $5 \mathrm{~dB}$. In general, the best improvements for both datasets are observed at 200$600 \mathrm{~m}$, which is expected as these regions show the strongest clutter power in the input channels (see clutter estimate in Fig. 8). In return, no improvement is obtained for depths where 'natural' nulls in the antenna patterns already prevent clutter returns, or where uniform weights generate a null in the clutter direction. In these cases the weights of the MVDR are equivalent to BS and thus the results are identical, explaining the coinciding curves at $\sim 340 \mathrm{~m}$ depth.

Regarding the differences between the two MVDR cases, ideally the optimum $\mathrm{CNR}_{0}$ should not vary with depth/range. However, in particular for depths below $600 \mathrm{~m}$, variable $\mathrm{CNR}_{0}$ values lead to the optimum suppression. This can be explained by imperfect modeling and poor estimation of the clutter angles. The main points are summarized as:

Non-nadir subsurface signal returns, i.e. reflections from the ice in off-nadir directions, which are received at the same time as the nadir echo and then scaled depending on the respective weighting coefficients. In particular, this explains the slightly higher returns for some MVDR solutions, compared with BS at shallow depths. Analyses show that in these regions the rise of the MVDR curve above the BS reference correlates with a stronger scaling of the non-nadir signal returns.

Suppression of clutter is optimized for dedicated angles rather than integration over the resolution cells. Hence, the total clutter power might not be optimally suppressed. The impact is higher for smaller bandwidths and 

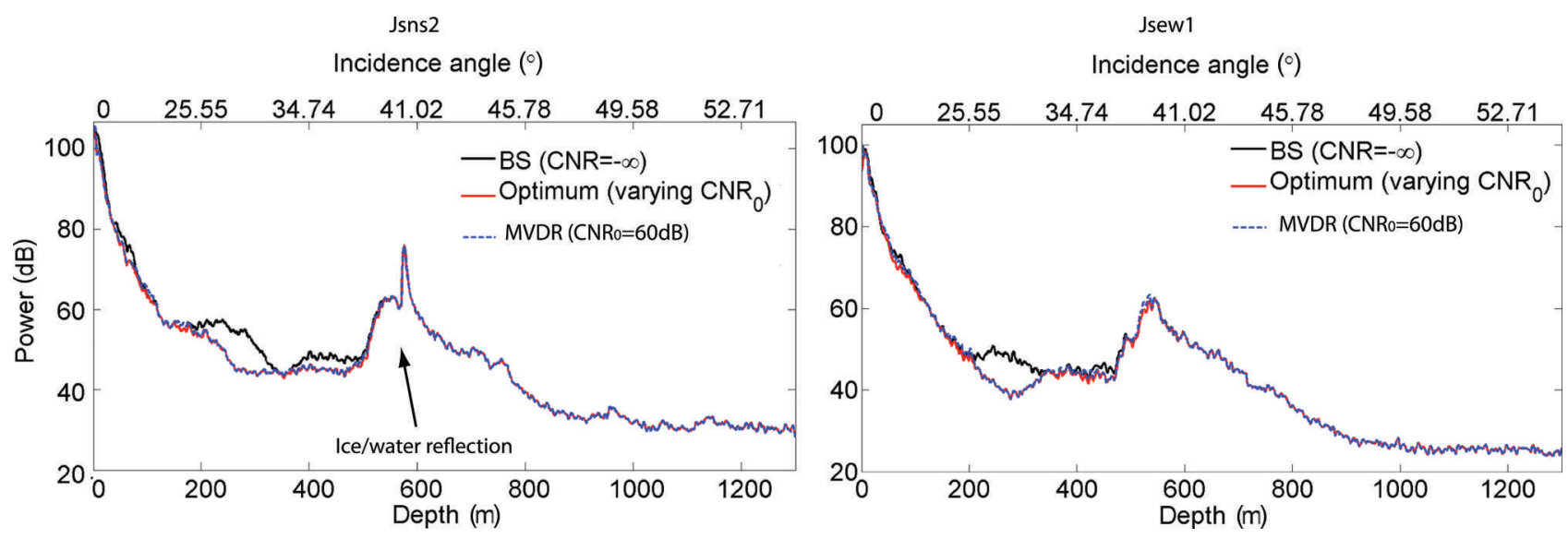

Fig. 14. Power profile $\mathrm{BB}^{\prime}$ for the Jsns2 track (left) and the Jsew1 track (right). BS (black) compared to MVDR (dashed blue) using a CNR ${ }_{0}$ of $60 \mathrm{~dB}$ for both the 6 and $85 \mathrm{MHz}$ bandwidths.

a possible solution could be to place broader nulls (e.g. in systems with more channels).

Channel imbalances, which can be accounted for by including the measured antenna channel patterns.

Estimation errors in clutter cell position. This is expected to improve by including accurate DEM information or estimation of the topography from the data themselves.

The dependency of the radar cross section of the ice surface on the incidence angle is not accurately known.

Mis-registration between channels for the larger bandwidths results in deteriorated clutter suppression.

These aspects will be investigated in detail in future work.

\section{The $B B^{\prime}$ profile}

For the $\mathrm{BB}^{\prime}$ profile, at the Jsns2 and Jsew1 track intersection, both $85 \mathrm{MHz}$ bandwidth datasets were acquired under similar conditions, with a difference of $4.6 \mathrm{~m}$ in altitude and $0.06^{\circ}$ in roll angle. Figure 14 reveals some differences between the profiles. Both tracks have the reflection of the ice/water interface at $\sim 550 \mathrm{~m}$ depth. For the Jsns 2 track there appears an additional peak. On average, the Jsns2 track appears to be shifted by $8 \mathrm{~dB}$. Part of this mismatch is explained by the calibration factor of $3.1 \mathrm{~dB}$, leaving a difference of $\sim 5 \mathrm{~dB}$. While the acquisition parameters are approximately the same, there is no knowledge of how the backscatter or the local across-track topography changes. A possible reason might be related to the null in nadir for the transmit antenna pattern, making the system gain highly sensitive to pointing. Another possibility might be a difference in ice crystal layering. For the ice stream, one could expect these layers to align with the flow direction. However, the impact should be rather subtle.

\section{SUMMARY AND DISCUSSION}

Different techniques for surface clutter suppression in ice sounding have been presented and compared by means of a sensitivity analysis. Beam steering optimizes the SNR without providing clutter suppression, while null steering provides optimum clutter reduction at the risk of a degraded SNR. The MVDR represents an attractive combination of beam steering and null steering, minimizing the joint power of clutter and white noise. This has been demonstrated to be particularly beneficial for sounding in the presence of grating lobes, and especially when the angular range of the grating lobes coincides with the bedrock. The limitation of this technique is that it requires data-dependent information on the CNR. The techniques investigated were applied to airborne multichannel icesounding data measured by POLARIS, acquired with both 6 and $85 \mathrm{MHz}$ bandwidths. The results confirmed the theoretical analyses and demonstrated successful clutter suppression with improvements of up to $10 \mathrm{~dB}$. A power profile comparison between the Jsns 2 and Jsew 1 tracks was made at their intersection over the floating glacier. While the main reflection occurs at a similar depth, the magnitude was found to be $\sim 5 \mathrm{~dB}$ larger for the Jsns2 track (corrected for calibration between both tracks). The reason for this remains unclear, but it might be partly attributed to the null in the transmit antenna pattern, making the gain highly sensitive to pointing variations around nadir. As the acquisition and processing parameters were approximately the same for both datasets, differences could be caused by a different backscatter variation in e.g. the direction parallel or perpendicular to the glacier flow, or from across-track topography variations.

The results allowed some aspects affecting the accuracy of the model that determines clutter suppression to be identified. Future improvements to this technique are suggested to include scaling of subsurface returns from offnadir directions, and incorporating information about the ice backscatter and surface topography. In addition, current techniques are optimized for dedicated directions of arrival only, which neglects the angular extension of clutter cells according to the resolution. Applying clutter-suppression techniques to multiple angular intervals represents an interesting approach for optimization. Finally, a peculiarity of the airborne geometry is that a bandwidth of $85 \mathrm{MHz}$ leads to stringent requirements regarding mis-registration between individual channels, restricting the applicability to relatively small angles. Additional topics for future work would be the incorporation of measured antenna patterns, including possible imbalances between the different channels. In addition, topographic information should be applied. These two aspects will enable estimation of the CNR to be further refined and thus lead to improved clutter suppression. 


\section{ACKNOWLEDGEMENTS}

We thank the members of the POLARIS Antarctic campaign team for organization and acquisition of data. We also acknowledge the German Aerospace research Center (DLR) for fruitful discussion, while performing a similar study in parallel for ESA (104671/11/NL/CT). Finally, we thank Hugh Corr, Anna Hogg and two anonymous reviewers for useful comments that helped improve the manuscript.

\section{REFERENCES}

Bekaert D (2012) Multi-phase center clutter suppression for synthetic aperture radar ice sounding. (ESTEC Technical Report Working Paper IPT-TN-ESA-335) European Space Agency, Noordwijk

Capon J (1969) High resolution frequency-wavenumber spectrum analysis. IEEE Proc., 57(8), 1408-1418 (doi: 10.1109/ PROC.1969.7278)

Dall J, Kristensen SS, Krozer V and Hernandez CC (2010) ESA'S POLarimetric Airborne Radar Ice Sounder (POLARIS): design and first results. Radar, Sonar Navig., IET, 4(3), 488-496 (doi: 10.1049/iet-rsn.2009.0035)

Dowdeswell JA and Evans S (2004) Investigations of the form and flow of ice sheets and glaciers using radio-echo sounding. Rep. Progr. Phys., 67(10), 1821-1861 (doi: 10.1088/0034-4885/67/ 10/R03)

European Space Agency (2013) The living planet programme. http:// www. esa. int/Our_Activities/Observing_the_Earth/The_Living Planet_Programme/ESA_s_Living_Planet_Programme

Fujita S, Matsuoka T, Ishida T, Matsuoka K and Mae S (2000) A summary of the complex dielectric permittivity of ice in the megahertz range and its applications for radar sounding of polar ice sheets. In Hondoh T ed. Physics of ice core records. Hokkaido University Press, Sapporo, 185-212

Gebert N, Krieger G and Moreira A (2009) Digital beamforming on receive: techniques and optimization strategies for high-resolution wide-swath SAR imaging. IEEE Trans. Aero. Electron. Syst., 45(2), 564-592 (doi: 10.1109/TAES.2009.5089542)

Guerci J (2003) Space-time adaptive processing for radar. Artech House, Norwood, MA

Jezek K and 7 others (2006) Glaciers and ice sheets mapping orbiter concept. J. Geophys. Res., 111(E6), E06S20 (doi: 10.1029/ 2005JE002572)

Jezek KC and 7 others (2011) Two-frequency radar experiments for sounding glacier ice and mapping the topography of the glacier bed. IEEE Trans. Geosci. Remote Sens., 49(3), 920-929 (doi: 10.1109/TGRS.2010.2071387)

Li J and 7 others (2013) High-altitude radar measurements of ice thickness over the Antarctic and Greenland ice sheets as a part of Operation IceBridge. IEEE Trans. Geosci. Remote Sens., 51(2), 742-754 (doi: 10.1109/TGRS.2012.2203822)
Nannini M, Prats P, Scheiber R, Villano M, Morrison K and Bennett J (2013) Multi phase center processing of ice sounding radar signals for across track surface clutter cancellation: comparison of surface clutter cancellation techniques and processor concept description. (DLR Monograph (Project Report) MPC104671-DD1-DLR) Deutsches Zentrum für Luft- und Raumfahrt, Oberpfaffenhofen

Nielsen U, Dall J, Kristensen SS and Kusk A (2012) Coherent surface clutter suppression techniques with topography estimation for multi-phase-center radar ice sounding. In Proceedings of 9th European Conference on Synthetic Aperture Radar (EUSAR 2012), 23-26 April, 2012, Nuremberg, Germany. Institute of Electrical and Electronics Engineers, Piscataway, NJ

Paden J, Allen C and Gogineni P (2010) 3D imaging of ice sheets. In Proceedings of IEEE International Geoscience and Remote Sensing Symposium (IGARSS 2010), 25-30 July 2010, Honolulu, HI, USA. Institute of Electrical and Electronics Engineers, Piscataway, NJ, 2611-2613

Peters ME, Blankenship DD, Carter SP, Kempf SD, Young DA and Holt JW (2007) Along-track focusing of airborne radar sounding data from West Antarctica for improving basal reflection analysis and layer detection. IEEE Trans. Geosci. Remote Sens., 45(9), 2725-2736 (doi: 10.1109/TGRS.2007.897416)

Rignot E, Mouginot J and Scheuchl B (2011) Antarctic grounding line mapping from differential satellite radar interferometry. Geophys. Res. Lett., 38(10), L10504 (doi: 10.1029/ 2011GL047109)

Scheiber R and Prats P (2007) Surface clutter suppression for ice sounding radars by coherent combination of repeat-pass data. In Proceedings of IEEE International Geoscience and Remote Sensing Symposium (IGARSS 2007), 23-28 July 2007, Barcelona, Spain. Institute of Electrical and Electronics Engineers, Piscataway, NJ, 3559-3562

Schmidt RO (1986) Multiple emitter location and signal parameter estimation. IEEE Trans. Antennas Propag., 34(3), 276-280 (doi: 10.1109/TAP.1986.1143830)

Stoica P and Nehorai A (1989) MUSIC, maximum likelihood, and Cramer-Rao bound. IEEE Trans. Acoust., Speech Signal Process., 37(5), 720-741 (doi: 10.1109/29.17564)

Stutzman WL and Thiele GA (1998) Antenna theory and design. Wiley, Hoboken, NJ

Velado B (2004) Radio echo sounding of Antarctica using a spaceborne P-band radar: from science to engineering. (ESTEC Technical Report Working Paper No. 2243) European Space Agency, Noordwijk

Villano M (2009) Synthetic aperture processing of ice sounding radar data: processing techniques and results from POLARIS proof-of-concept campaign. (ESTEC Technical Report Working Paper 2356) European Space Agency, Noordwijk

Wu X, Jezek KC, Rodriguez E, Gogineni S, Rodriguez-Morales F and Freeman A (2011) Ice sheet bed mapping with airborne SAR tomography. IEEE Trans. Geosci. Remote Sens., 49(10 Pt 1), 3791-3802 (doi: 10.1109/TGRS.2011.2132802) 